\title{
Magneto-optical Spectra of Pt/Co GMR Structures with Capping Layers
}

\author{
T. Ishibashi, M. Naganuma, S. Tang, S. Goto, K. Machida*, K. Furukawa**, K. Aoshima*, \\ N. Funabashi*, K. Kuga*, H. Kikuchi* and N. Shimidzu* \\ Nagaoka University of Technology, 1603-1 Kamitomioka, Nagaoka, Niigata 940-2188, Japan \\ *NHK Science \& Technology Research Laboratories, 1-10-11 Kinuta, Setagaya-ku, Tokyo \\ **Tokyo Denki Univ., 2-2 Kanda-Nishiki-cho, Chiyoda-ku, Tokyo 101-8457, Japan
}

The MO spectra of GMR structures with capping layers, $\mathrm{Si} / \mathrm{SiO}_{2} /$ bottom electrode/TbFeCo $(9 \mathrm{~nm}) / \mathrm{Cu}(4 \mathrm{~nm}) / \mathrm{Co}$ $(0.1 \mathrm{~nm}) / \mathrm{Pt}(1.1 \mathrm{~nm}) /(\mathrm{Pt}(1.1 \mathrm{~nm}) / \mathrm{Co}(6 \mathrm{~nm}) \times 4) / \mathrm{cap}(\mathrm{cap}=\mathrm{Ta}$, Au, Pt $(3 \mathrm{~nm}))$, were studied. We found that the optical constants and Kerr spectra exhibited different behaviors for all samples. The differences in the optical constants of these samples were analyzed by means of optical simulation. The MO spectrum is discussed in terms of simulation carried out by the virtual optical constant method for $(\mathrm{PtCo}) / \mathrm{cap}(\mathrm{cap}=\mathrm{Ta}, \mathrm{Au}, \mathrm{Pt}(3 \mathrm{~nm}))$.

Key words: magneto-optical spectrum, GMR, capping layer, optical constant, $\mathrm{Pt} / \mathrm{Co}$, PtCo

\section{Introduction}

Optical modulating devices with high-speed switching times and high resolutions of pitch of less than $1 \mu \mathrm{m}$ are required to achieve ultimate three-dimensional displays utilizing holography. Spintronic devices, such as giant magnetoresistance (GMR) and tunnel magnetoresistance (TMR) devices, driven by spin transfer switching are promising candidates because these devices are capable of high-speed operation with their small size of less than 1 $\mu \mathrm{m}^{1)}$. However, the magneto-optical (MO) effect, i.e., Kerr rotation or Kerr ellipticity, utilized to modulate light intensity is usually smaller than $1^{\circ}$ even for MO materials, while liquid crystal devices achieve $90^{\circ}$ for the rotation angle of polarization. In addition, these devices have complicated structures, e.g., consisting of a bottom electrode, pinned layer, nonmagnetic layer, free layer, capping layer, and top electrode, and their thickness is thin enough for visible light to pass through them. Therefore, the MO effect of these devices has to be investigated to attain sufficient optical modulation. We have already reported that $3-\mathrm{nm}$-thick capping layers affect the MO spectra and optical properties of GMR structures ${ }^{2}$. This paper reports the analysis of optical constants and MO spectra of GMR structures with different capping materials.

\section{Experiments}

A Pt/Co GMR structure, $\mathrm{Si} / \mathrm{SiO}_{2} /$ bottom electrode/TbFeCo $(9 \mathrm{~nm}) / \mathrm{Cu}(4 \mathrm{~nm}) / \mathrm{Co}(0.1 \mathrm{~nm}) / \mathrm{Pt}(1.1$ $\mathrm{nm}) /(\mathrm{Pt} \quad(1.1 \mathrm{~nm}) / \mathrm{Co}(6 \mathrm{~nm}) \times 4)$, with perpendicular magnetic anisotropy was deposited on a $\mathrm{Si} / \mathrm{SiO}_{2}$ substrate by DC magnetron sputtering with a base pressure of less than $2 \times 10^{-6} \mathrm{~Pa}$. The film was annealed in vacuum at $190^{\circ} \mathrm{C}$ for an hour in the absence of a magnetic field. The magnetic properties of the films were not expected to change after a dry photoresist process at $190^{\circ} \mathrm{C}$ due to this annealing process. Finally, a capping layer $(\mathrm{Ta}, \mathrm{Au}, \mathrm{Pt})$ with a thickness of $3 \mathrm{~nm}$ was deposited on top of the films.

The Kerr rotation spectra were measured by an MO spectrometer using the polarization modulation technique with a wavelength between 260-900 $\mathrm{nm}$. MO spectra were obtained as an average of two spectra measured with magnetic fields of $\pm 2 \mathrm{kOe}$ to measure the Kerr rotation of the free layers, which is sufficiently higher than the coercivity of the free layers $(\sim 1 \mathrm{kOe})$ and lower than that of the pinned layers $(>5 \mathrm{kOe})$.

Optical constants, refractive indices $n$ and extinction coefficients $k$, were measured by using an ellipsometer (Horiba Jobin Yvon: MM-16) with a wavelength between 430-700 nm, and these were analyzed by optical simulation using software (Horiba: Delta $\mathrm{Psi}^{2}$. The structure of a $\mathrm{Si}$ sub./ $\mathrm{SiO}_{2} / \mathrm{X} /$ capping layer was assumed in order to simplify the optical analysis. The optical constants of the $\mathrm{X}$ layer consisting of the bottom electrode/TbFeCo $(9 \mathrm{~nm}) / \mathrm{Cu}(4$ $\mathrm{nm}) / \mathrm{Co}(0.1 \mathrm{~nm}) / \mathrm{Pt}(1.1 \mathrm{~nm}) /(\mathrm{Pt}(1.1 \mathrm{~nm}) / \mathrm{Co}(6 \mathrm{~nm}) \times 4)$ were deduced from that of the GMR film with the $\mathrm{Pt}$ capping layer by using the ellipsometer ${ }^{2}$. The optical constants of $\mathrm{Au}, \mathrm{Ta}$, and $\mathrm{Pt}$ used in the optical analysis were measured for each film deposited on the $\mathrm{Si}$ substrate with the ellipsometer, and those of $\mathrm{Si}$ and $\mathrm{SiO}_{2}$ were obtained from a published report ${ }^{3)}$.

The magneto-optical spectra were calculated with the virtual optical constant method ${ }^{4}$, where the capping layer with complex refractive index $\hat{n}_{2}$ and thickness $d_{2}$ on the PtCo alloy with $\hat{n}_{1}^{ \pm}$and infinite thickness were replaced by a bulk material with virtual optical constant $\hat{N}^{ \pm}$, 


$$
\hat{N}^{ \pm}=\hat{n}_{2} \frac{1-\hat{r}^{ \pm} \exp \left(-2 i \phi^{ \pm}\right)}{1+\hat{r}^{ \pm} \exp \left(-2 i \phi^{ \pm}\right)}
$$

Here, $\hat{n}_{1}^{ \pm}=n_{1}^{ \pm}-i k_{1}^{ \pm}, \quad \hat{n}_{2}=n_{2}-i k_{2}, \quad \hat{r}^{ \pm}=\left(\hat{n}_{2}-\hat{n}_{1}^{ \pm}\right) /\left(\hat{n}_{2}+\hat{n}_{1}^{ \pm}\right)$, and $\phi^{ \pm}=2 \pi \hat{n}_{2} d_{2} / \lambda$, where the signs \pm denote right and left circular polarizations. Kerr rotation and ellipticity are given as the real and the imaginary parts of the complex by

$$
\begin{aligned}
& \theta_{k}=\operatorname{Re}\left\{\hat{E}_{x y} / \hat{N}\left(1-\hat{N}^{2}\right)\right\} \text { and } \\
& \eta_{k}=\operatorname{Im}\left\{\hat{E}_{x y} / \hat{N}\left(1-\hat{N}^{2}\right)\right\},
\end{aligned}
$$

where $\hat{N}=\left(\hat{N}^{+}+\hat{N}^{-}\right) / 2, \hat{E}_{X Y}=\left(\hat{N}^{+^{2}}-\hat{N}^{-2}\right) / 2 i$.

A model consisting of a capping layer and PtCo bulk was used in this study. The reason we used PtCo alloy instead of a Pt/Co multilayer was that PtCo alloy has similar optical properties to those of the $\mathrm{Pt} / \mathrm{Co}$ multilayer, which we did not have.

\section{Results and discussion}

\subsection{Optical constants of GMR structures}

Figure 1 shows the refractive indices and extinction coefficients for three samples with different capping layers $(\mathrm{Ta}, \mathrm{Au}$, and $\mathrm{Pt}$ ) measured by means of ellipsometry. We found that they had different values and structures, although the main difference was only in the kinds of materials used for the 3-nm-thick capping layers. To find what effects the capping layers had, we analyzed these spectra as the samples consisted of a $\mathrm{Si} / \mathrm{SiO}_{2} / \mathrm{X}$ (bottom electrode/ $\mathrm{TbFeCo} / \mathrm{Cu}$ )/capping layer.

The optical constants of the GMR structures were reconstructed from the simulation using these $\mathrm{Si}, \mathrm{SiO}_{2}$, $\mathrm{X}$ elements obtained from the GMR structure with the $\mathrm{Pt}$ capping layer, and the $\mathrm{Ta} / \mathrm{Ta}_{2} \mathrm{O}_{5}, \mathrm{Pt}, \mathrm{Au}$ and $\mathrm{Ta}$ capping layer as seen in Fig. 2. The Ta capping layer does not correspond to the experimental results. However, this agrees well with the experimental data in Fig. 1 if oxidization in the upper part of the Ta layer is assumed, where the thickness of Ta is $0.95 \mathrm{~nm}$ and that of and $\mathrm{Ta}_{2} \mathrm{O}_{5}$ is $2.1 \mathrm{~nm}$. Consequently, we found that the differences in the optical constants of the GMR structure with the capping layers could be explained by using the optical constants of the capping layers.

Figure 3 shows the Kerr rotation spectra of free layers with different capping layers of $\mathrm{Ta}, \mathrm{Au}$, and $\mathrm{Pt}$, which has been reported ${ }^{2}$, and Figure 4 shows the Kerr ellipticity of the same samples. Features that peak around $4 \mathrm{eV}$ in Kerr rotation and those that increase with photon energy in Kerr ellipticity are similar to those in $\mathrm{Pt} / \mathrm{Co}$ multilayers previously reported ${ }^{5,6}$. These results indicate that the Kerr spectra in Figs. 3 and 4 have characteristics of the $\mathrm{Pt} / \mathrm{Co}$ multilayer used as free layers. However, we found that the values for Kerr rotation and peak positions seemed to depend on the capping layer materials. The peak positions of those spectra are 4.1, 4.3, and $3.8 \mathrm{eV}$, and the values of maximum Kerr rotation are 0.35, 0.25, and 0.23 degrees for samples with $\mathrm{Ta}, \mathrm{Au}$, and $\mathrm{Pt}$ capping layers, respectively. Kerr ellipticity also seems to depend on the materials for the capping layers.

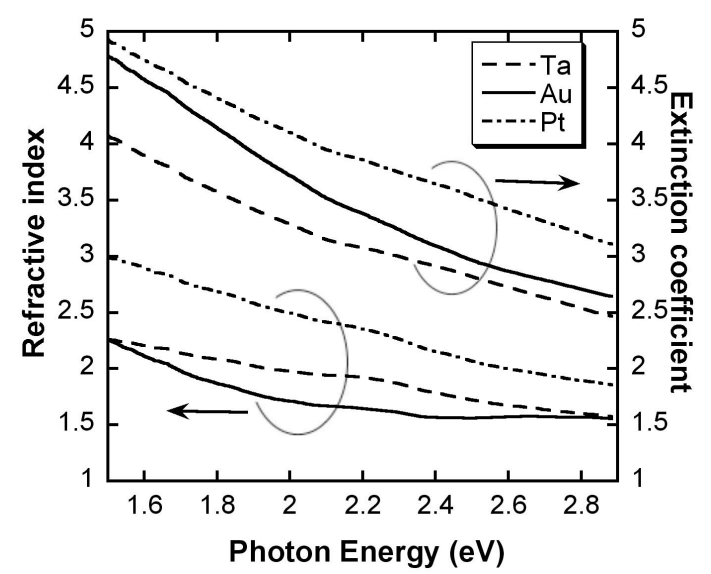

Fig. 1 Optical constants of GMR films with capping layers of $\mathrm{Ta}, \mathrm{Au}$, and $\mathrm{Pt}$.

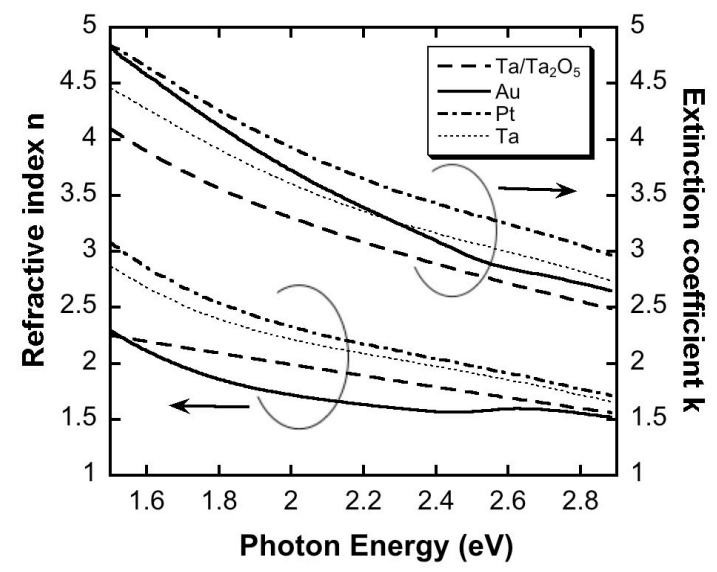

Fig. 2 Simulated optical constants of GMR films with capping layers of $\mathrm{Ta}, \mathrm{Au}$, and $\mathrm{Pt}$.

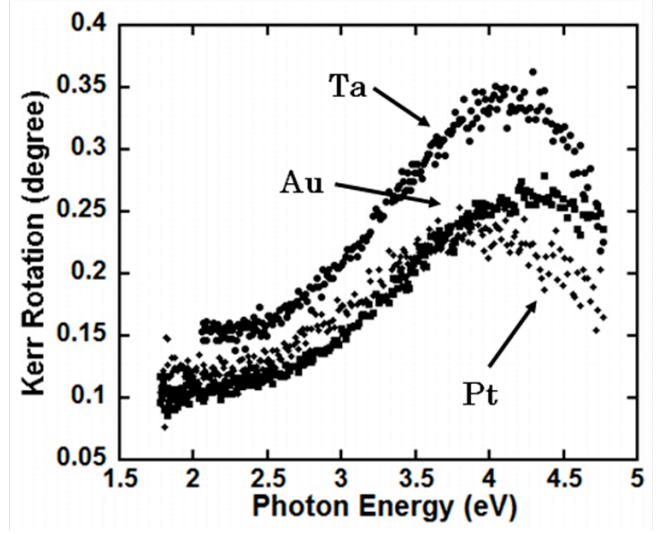

Fig. 3 Kerr rotation spectra of GMR films with capping layers of Ta, Au and Pt. 


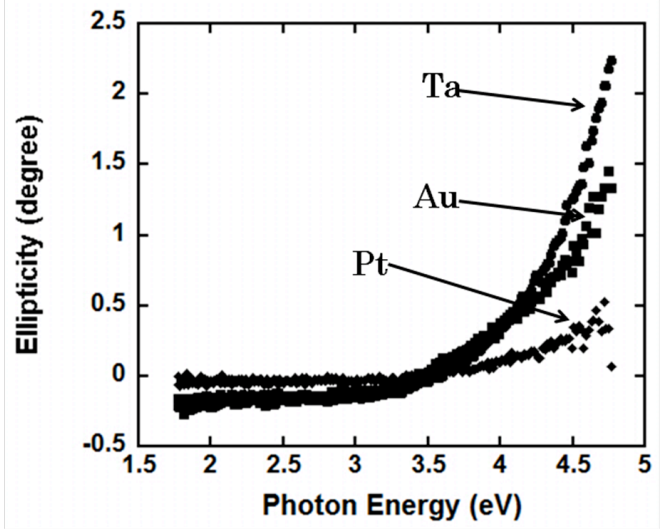

Fig. 4 Kerr ellipticity spectra of GMR films with capping layers of $\mathrm{Ta}, \mathrm{Au}$ and $\mathrm{Pt}$.

To better understand the differences in Kerr rotation and Kerr ellipticity, the MO spectra of the PtCo alloy with a capping layer of $\mathrm{Au}, \mathrm{Pt}, \mathrm{Ta}$, and $\mathrm{Ta}(0.9$ $\mathrm{nm}) / \mathrm{Ta}_{2} \mathrm{O}_{5}(2.1 \mathrm{~nm})$ with a thickness of $3 \mathrm{~nm}$ were calculated using the virtual optical constant method. The calculated Kerr rotation and Kerr ellipticity are shown in Figs. 5 and 6 . The values of Kerr rotation for $\mathrm{PtCo} / \mathrm{Ta} / \mathrm{Ta}_{2} \mathrm{O}_{5}$ are larger than those for $\mathrm{PtCo}$ with higher photon energies above $3.5 \mathrm{eV}$, while those for $\mathrm{Pt}$, $\mathrm{Au}$, and $\mathrm{Ta}$ are smaller than those for $\mathrm{Pt} \mathrm{Co} / \mathrm{Ta} / \mathrm{Ta}_{2} \mathrm{O}_{5}$ between 1.5 and $5 \mathrm{eV}$. In addition, the values for the ellipticity of $\mathrm{PtCo} / \mathrm{Ta} / \mathrm{Ta}_{2} \mathrm{O}_{5}$ are higher than those for $\mathrm{Pt}$, $\mathrm{Au}$, and Ta above $3.5 \mathrm{eV}$. However, the values for both Kerr rotation and Kerr ellipticity for $\mathrm{PtCo} / \mathrm{Ta}$ are smaller than those for $\mathrm{PtCo} / \mathrm{Ta} / \mathrm{Ta}_{2} \mathrm{O}_{5}$ and do not agree with the measured data in Fig. 3, indicating that the surface of Ta is oxidized as previously mentioned.

The values for calculated Kerr rotation For $\mathrm{PtCo} / \mathrm{Au}$ are larger than those for $\mathrm{PtCo} / \mathrm{Pt}$, while the values for both calculated Kerr ellipticity spectra are similar. The results for Kerr rotation are consistent with the experimental data in Fig. 3. However, the relative peak positions in the Kerr rotation spectra are different, i.e., the peak position for $\mathrm{PtCo} / \mathrm{Au}$ is lower than that for $\mathrm{PtCo} / \mathrm{Pt}$ in Fig. 3, and their relation is opposite in Fig. 5. The reason that $\mathrm{PtCo} / \mathrm{Au}$ and $\mathrm{PtCo} / \mathrm{Pt}$ have similar ellipticity spectra is because the optical constants, $n$ and $k$, of $\mathrm{Au}$ and Pt have similar values for a photon energy from $4-5 \mathrm{eV}$.

The peak positions in the Kerr spectra could not be reproduced in our simulation and the ellipticity spectra could not be fully explained. This suggests that $\mathrm{Au}, \mathrm{Pt}$, or Ta affects the electronic structure of PtCo underneath the capping layer. We believe that this can happen, since it is known that the metallic multilayer has a peculiar electronic structure that cannot be merely explained by assuming the stack of metals observed in $\mathrm{Pd} / \mathrm{Co}$ and $\mathrm{Fe} / \mathrm{Au}$ multilayers ${ }^{7,8)}$.

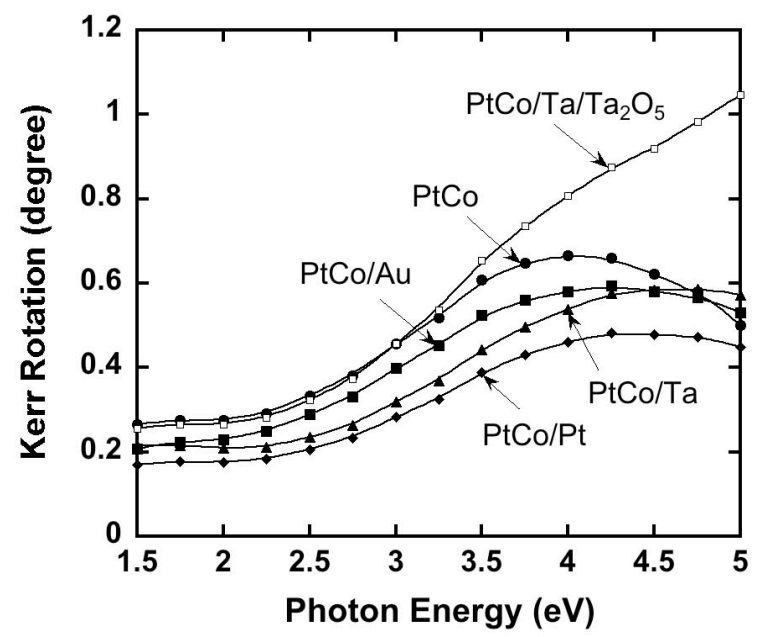

Fig. 5 Kerr rotation spectra calculated for PtCo alloy and other alloys with capping layers.

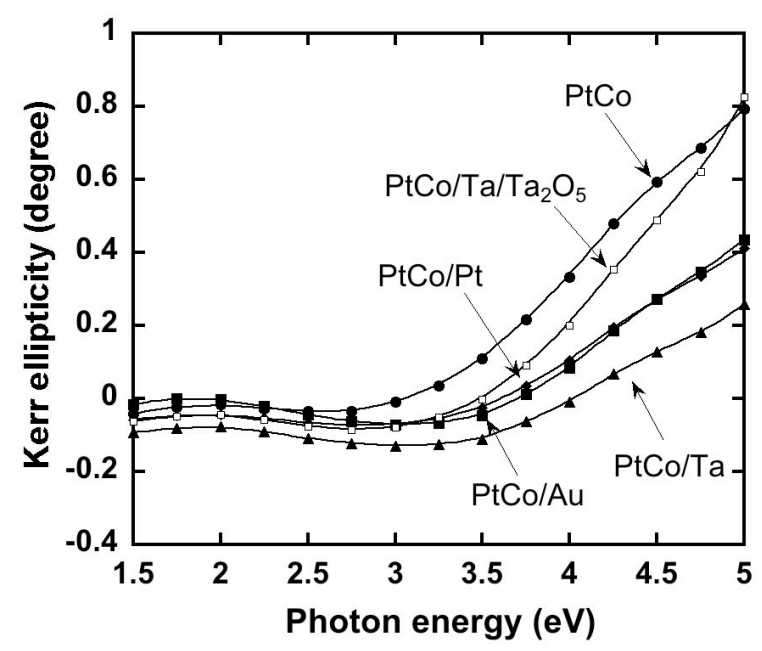

Fig. 6 Kerr ellipticity spectra calculated for PtCo alloy and other alloys with capping layers.

\section{Summary}

MO and optical properties of the GMR structures with different capping layers with a thickness of $3 \mathrm{~nm}$ were studied. The optical simulation was revealed that the optical constants of the GMR structures could be explained by using those of the capping layers of $\mathrm{Au}$ and Pt. For Ta, the calculated spectrum agreed with the experimental data by assuming an oxidation of Ta. For MO spectra, the simulation reproduced that the large Kerr rotation for Ta capping layer by assuming the surface of Ta was oxidized and the small Kerr rotation for Pt capping layer. On the other hand the peak shift in Kerr rotation spectra could not be explained.

Acknowledgements This research was supported by the National Institute of Information and Communications Technology (NICT). 


\section{References}

1) K.Aoshima, N. Funabashi, K. Machida, Y. Miyamoto, N. Kawamura, K. Kuga, N. Shimidzu, F. Sato, T. Kimura, Y.Ohtani, Appl. Phys. Lett., 91, 052507, (2007).

2) T. Ishibashi, M. Naganuma, T. Honma, T. Komatsu, K. Machida, K. Furukawa, K. Aoshima, N. Funabashi, K. Kuga, N. Shimidzu, J. Phys.: Conf. Series, (2011) (to be published). 3) G.E.Jellison, Jr., Optical Materials, 1, 41 (1992).

4) K. Ohta, A. Takahashi, T. Deguchi, T. Hyuga, S. Kobayashi, H. Yamaoka, SPIE 382, 252 (1983).
5) W. B. Zeper, F. J. A. M. Greidanus, P. F. Carcia, C. R. Fincher, J. Appl. Phys., 65, 4971 (1989).

6) K. Sato, H. Hongu, H. Ikekame, J. Watanabe, K. Tsuzukiyama, Y. Togami, M. Fujisawa, T. Fukazawa, Jpn. J. Appl. Phys., 31, 3603 (1992).

7) Y. Tosaka, H. Ikekame, K. Urago, S. Kurosawa, K. Sato, S. C. Shin, J. Magn. Soc. Jpn., 18, 389 (1994).

8) K. Sato, E. Takeda, M. Akita, M. Yamaguchi, K. Takanashi, S. Mitani, H. Fujimori, Y. Suzuki, J. Magn. Magn. Mater., 18, 177 (1998).

Received June 18, 2011; Accepted September 6, 2011 